\title{
High gain proportional rf control stability at TESLA cavities
}

\author{
Elmar Vogel \\ Deutsches Elektronen Synchrotron (DESY), Notkestraße 85, 22607 Hamburg, Germany
}

(Received 1 February 2007; published 3 May 2007)

\begin{abstract}
Fast proportional rf control is used as the basis for rf field regulation in actual linear accelerator projects like the international linear collider (ILC) and the European X-ray free electron laser (XFEL) based on TESLA technology. Additional control loops improve the field regulation by treating repetitive effects and compensating the beam loading. Nevertheless, the ability for high gain operation of the fast loops is desirable for the strong suppression of nonpredictive and nonrepetitive disturbances. TESLA cavities host nine fundamental modes (FMs) where only one is used for beam acceleration. The unwanted FMs have a significant influence on the proportional rf control loop stability at high gains. Within this paper, the stability of proportional rf control loops taking the FMs and digitalization effects into account will be discussed in detail together with measures enabling a significant increase of the gain values.
\end{abstract}

DOI: 10.1103/PhysRevSTAB.10.052001

PACS numbers: 29.17.+w

\section{INTRODUCTION}

Recent linear accelerator projects such as the international linear collider (ILC) and the European x-ray free electron laser (XFEL) are based on the superconducting rf technology whose development was started by the teraelectronvolt superconducting linear accelerator (TESLA) collaboration. High accelerating gradients at moderate cryogenic load are obtained by a pulsed operation with repetition frequencies from $5 \mathrm{~Hz}$ up to $30 \mathrm{~Hz}$ and keeping the accelerating gradients for $800 \mu$ s at constant levels of up to $30 \mathrm{MV} / \mathrm{m}$ and more (at the ILC). For economic reasons, one high power klystron will drive 32 or more superconducting $1.3 \mathrm{GHz}$ cavities.

The requirements on the accelerating rf fields vary slightly from project to project. The ILC is focused on the highest gradients possible whereas FELs have extreme demands on the rf field stability required for effective bunch compression. Altogether, the use of digital rf control is beyond question due to its flexibility concerning the multicavity control, the adoptability to changing requirements, and enabling new rf control concepts. Above all, digital controllers are more suitable for automation and exception handling.

Fast proportional $\mathrm{rf}$ control is used as the basis for the $\mathrm{rf}$ field regulation [1]. Additional control loops are built up around the fast loops, improving the field regulation by treating repetitive effects and compensating the beam loading $[2,3]$. Nevertheless, the ability for high gain operation of the fast loops is desirable for strong suppression of nonpredictive and nonrepetitive disturbances.

TESLA cavities consist of nine cells and therefore host nine fundamental modes (FMs) where only one is used for beam acceleration [4]. The other FMs are unwanted and have a significant influence on the proportional control loop stability at high gains [1,5]. Digitalization acts like a filter and therefore influences the loop stability. In the literature, digitalization effects at accelerator rf control have not yet been discussed in detail. In this paper, the stability of proportional single input single output (SISO)type rf control loops at TESLA cavities taking the FMs and the digitalization into account will be discussed together with measures allowing for high gain values.

\section{FUNDAMENTAL MODES OF TESLA CAVITIES}

The superconducting TESLA cavities are each composed of nine cells with a length of one-half of the $1.3 \mathrm{GHz}$ wavelength (Fig. 1). The rf power is fed into the cavity via an rf power coupler located near the end cell at one side of the cavity. Coupling from cell to cell leads to a build up of standing rf waves.

An rf cavity consisting of nine cells hosts nine different normal rf modes and fundamental modes (FMs), respectively, with electrical field vectors on the cavity axis parallel to it $\left(\mathrm{TM}_{010}\right.$ modes). The set of the nine amplitudes of the electrical field vectors in the corresponding cells can be described by "amplitude" vectors $\mathbf{V}$ consisting of nine elements. Using symmetry arguments and group theory [6-8], one immediately realizes the nine modes can be described by a set of nine orthogonal amplitude vectors with each vector symmetrical to a plane perpendicular to

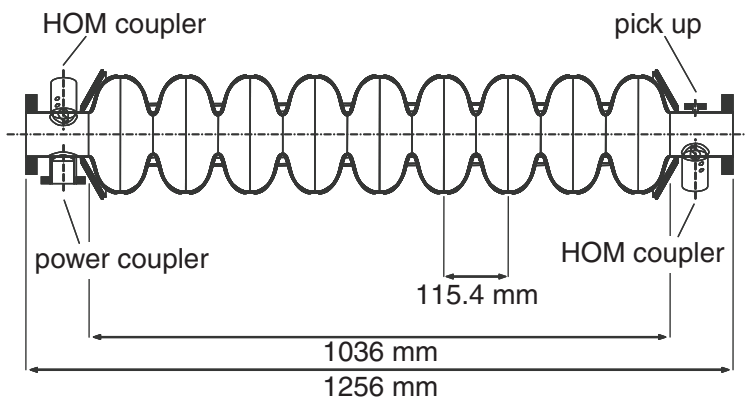

FIG. 1. Side view of a nine cell TESLA type cavity with the main power coupler, the pickup probe, and two higher order mode (HOM) couplers. 
the longitudinal cavity axis centered in the middle cell

$$
\mathbf{V}_{(n / 9) \pi}=\sqrt{\frac{2}{9}}\left(\begin{array}{c}
\sin \left((1-1 / 2) \frac{n}{9} \pi\right) \\
\sin \left((2-1 / 2) \frac{n}{9} \pi\right) \\
\vdots \\
\sin \left((9-1 / 2) \frac{n}{9} \pi\right)
\end{array}\right) \text { for } n \neq 9
$$

and

$$
\mathbf{V}_{\pi}=\frac{1}{\sqrt{9}}\left(\begin{array}{c}
1 \\
-1 \\
1 \\
-1 \\
\vdots \\
1
\end{array}\right)
$$

In accelerator technology, one names the modes in correspondence to the rf field amplitude phase advance within each cell, which, in the case of TESLA cavities, is the factor $\frac{n}{9} \pi$. For acceleration only the $\frac{9}{9} \pi=\pi$-mode is used, the other modes are unwanted. Figure 2 shows a sketch of the arrangements of the rf field amplitudes for all nine FMs. The $\pi$-mode shows equal rf field amplitudes in all cells with opposite sign. While the beam passes from cell to cell, the sign of the rf field changes synchronously leading to beam acceleration over the full cavity length. This is not the case for the other modes.

The action of the coupling from cell to cell is strongest when subsequent cells have opposite field vector sign ( $\pi$-mode), whereas weakest in case they point in the same direction $\left(\frac{1}{9} \pi\right.$-mode). Obviously, this results in the highest eigenfrequency for the $\pi$-mode and the lowest for the $\frac{1}{9} \pi$-mode (Table I).

The unloaded quality factors of all nine modes are equal $[10,11]$ with values of $Q_{0} \approx 10^{10}$. This is not the case for the loaded quality factors $Q_{L}$ determined by the coupling $\beta$.

TABLE I. Frequencies of the FMs measured in the superconducting state at 116 cavities produced for the TESLA collaboration and succeeding projects [9]. The values are the mean eigenfrequency and the rms of the eigenfrequencies (not the mode bandwidths).

\begin{tabular}{cl}
\hline \hline FM & Mean eigenfrequency of 116 cavities \\
\hline$\pi$ & $f_{\pi}=(1300444 \pm 303) \mathrm{kHz}$ \\
$8 / 9 \pi$ & $f_{\pi}-(785 \pm 51) \mathrm{kHz}$ \\
$7 / 9 \pi$ & $f_{\pi}-(3053 \pm 94) \mathrm{kHz}$ \\
$6 / 9 \pi$ & $f_{\pi}-(6501 \pm 157) \mathrm{kHz}$ \\
$5 / 9 \pi$ & $f_{\pi}-(10694 \pm 243) \mathrm{kHz}$ \\
$4 / 9 \pi$ & $f_{\pi}-(15122 \pm 347) \mathrm{kHz}$ \\
$3 / 9 \pi$ & $f_{\pi}-(19237 \pm 430) \mathrm{kHz}$ \\
$2 / 9 \pi$ & $f_{\pi}-(22594 \pm 503) \mathrm{kHz}$ \\
$1 / 9 \pi$ & $f_{\pi}-(24773 \pm 543) \mathrm{kHz}$ \\
\hline \hline
\end{tabular}

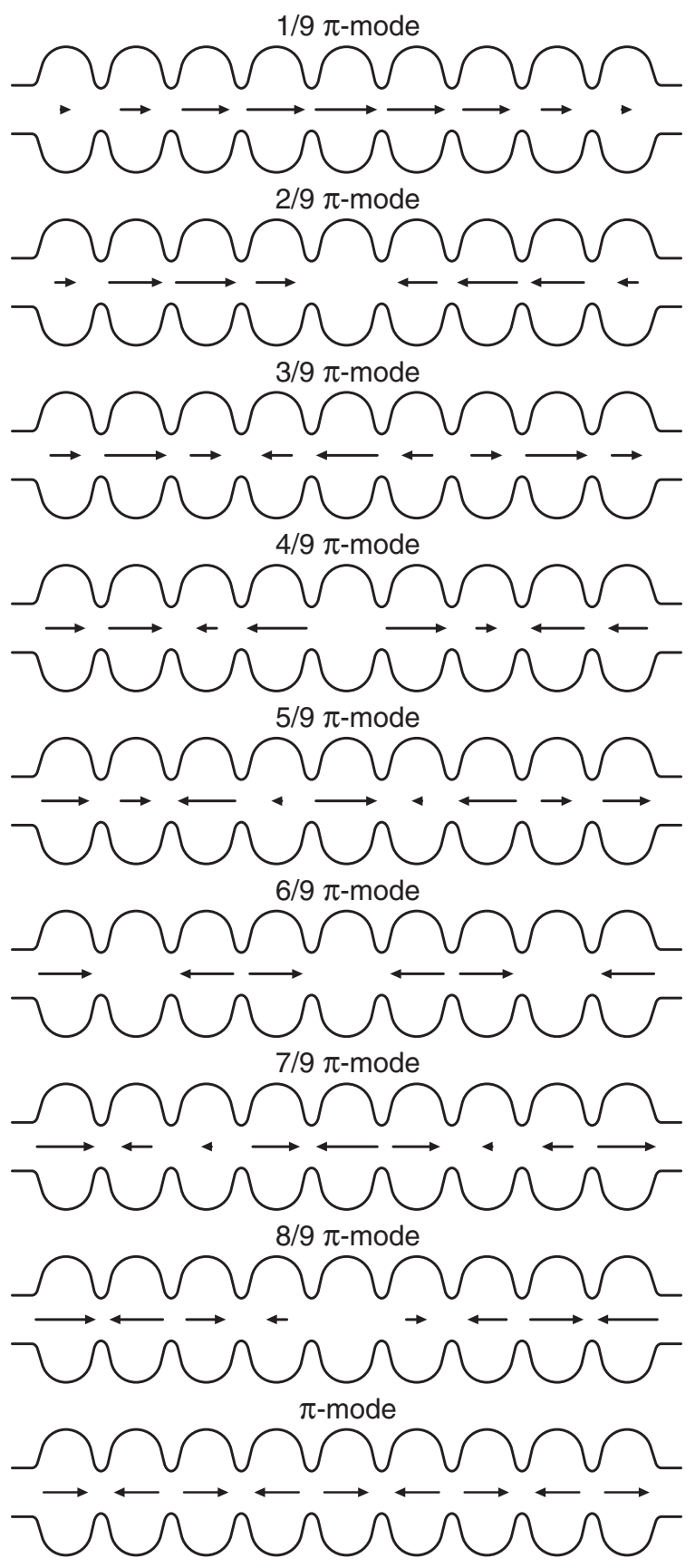

FIG. 2. The rf field amplitudes of the normal and fundamental modes (FMs) in 9-cell cavities.

Differences in the field amplitudes of the end cells, most visible at the $\frac{1}{9} \pi$-mode (Fig. 2), lead to differences in the coupling to the power coupler and the pickup, respectively. For instance, the $\frac{1}{9} \pi$-mode is least coupled to the power coupler and shows consequently the highest loaded quality factor. For the $\pi$-mode, the loaded quality factor has values of $Q_{L \pi} \approx 3 \times 10^{6}$. Coupling and loaded quality factors for the other modes can be estimated as follows.

The electromagnetic energy contained in a mode of a nine cell cavity is proportional to the scalar product 


$$
\mathbf{V}_{(n / 9) \pi} \mathbf{V}_{(n / 9) \pi}=\sum_{i=1}^{9}\left(V_{(n / 9) \pi}\right)_{i}^{2}
$$

Because of the normalization (1) and (2), the vectors $\mathbf{V}_{(n / 9) \pi}$ describe modes containing the same amount of stored energy. In contrast, the energy contained in the end cells varies with $\left(V_{(n / 9) \pi}\right)_{1}^{2}$. The coupling to the modes is to the coupling of the $\pi$-mode as the energies in the end cells is to the energy in the end cell at the $\pi$-mode [12]. Hence, we obtain

$$
\frac{\beta_{(n / 9) \pi}}{\beta_{\pi}}=2 \sin ^{2} \frac{n \pi}{18}
$$

From the relation between the loaded quality factor and coupling [1],

$$
Q_{L}=\frac{Q_{0}}{1+\beta} \approx \frac{Q_{0}}{\beta} \quad \text { approximation for } Q_{0} \gg Q_{L},
$$

we get

$$
\frac{Q_{L(n / 9) \pi}}{Q_{L \pi}}=\frac{\beta_{\pi}}{\beta_{(n / 9) \pi}}=\left(2 \sin ^{2} \frac{n \pi}{18}\right)^{-1} .
$$

For the numerical values of $\beta_{(n / 9) \pi}$ and $Q_{L(n / 9) \pi}$ normalized to the values for the $\pi$-mode, see Table II.

In addition to the FMs, TESLA type cavities also host modes with electrical field vectors on the cavity axis with either tiny amplitudes parallel to it or with considerable amplitudes but perpendicular to the cavity axis (modes of the TE/TM and TM/TE pass bands [10]). The frequencies of these higher order modes (HOMs) range from 1.62 to $1.89 \mathrm{GHz}[10,11]$. For acceleration they are unwanted and are therefore damped via HOM couplers. In this paper they are not further considered as their frequencies are far away from the operational frequency and in addition are outside the bandwidth of the high power rf.

TABLE II. List of coupling factors and loaded quality factors of the FMs.

\begin{tabular}{ccc}
\hline \hline FM & $\beta / \beta_{\pi}$ & $Q_{L} / Q_{L \pi}$ \\
\hline$\pi$ & 1.0000 & 1.000 \\
$8 / 9 \pi$ & 1.9397 & 0.516 \\
$7 / 9 \pi$ & 1.7660 & 0.566 \\
$6 / 9 \pi$ & 1.5000 & 0.667 \\
$5 / 9 \pi$ & 1.1736 & 0.852 \\
$4 / 9 \pi$ & 0.8264 & 1.210 \\
$3 / 9 \pi$ & 0.5000 & 2.000 \\
$2 / 9 \pi$ & 0.2340 & 4.274 \\
$1 / 9 \pi$ & 0.0603 & 16.582 \\
\hline \hline
\end{tabular}

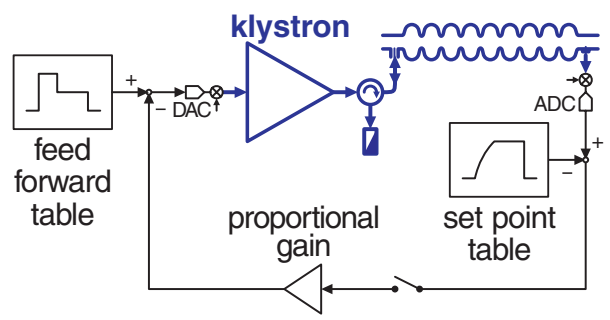

FIG. 3. (Color) Sketch of a pulsed rf superconducting cavity control loop. DACs in front of the vector modulator $(\otimes)$ and ADCs after down converters $(\otimes)$ are part of the digital controller (black).

\section{PROPORTIONAL CONTROL STABILITY IN GENERAL}

Figure 3 shows a sketch of a digital proportional control loop, regulating $\mathrm{rf}$ amplitude and phase, and angular pointer components (IQ), respectively, within a superconducting nine cell cavity driven by a pulsed klystron. For simplification most additional technical components such as the klystron preamplifier are omitted. Because of the narrow bandwidth of the cavity, the rf drive voltage supplied by the klystron results in a slow voltage increase inside the cavity while filling. When reaching the gradient desired, the beam starts passing the cavity. With nominal beam current, the gradient reduction due to beam loading is exactly compensated by the rf drive voltage resulting in a constant gradient level applied to the beam (flattop). In the case of beam current below nominal, the drive voltage has to be reduced after filling to keep the cavity voltage constant. For error signal calculation, the slow filling is taken into account using an appropriate set point table [1]. At the end of flattop both the $\mathrm{rf}$ drive and control loop are switched off.

For initial loop stability examinations, one can investigate "Bode plots" $[13,14]$ of the continuous open loop transfer function, keeping in mind the "Nyquist stability criterion" $[13,14]$ has to be applied instead in case of doubt (as discussed later). Within this section, digitalization effects are neglected. Then, the IQ transfer function of the loop is composed of the klystron (high power rf) transfer function, the cavity transfer function, the proportional gain factor, and the loop delay.

More detailed examinations of $1.3 \mathrm{GHz}$ klystron properties measuring the frequency response over larger ranges of difference frequencies have recently been performed [15]. Klystrons from different manufacturers show quite distinct frequency spectra at larger difference frequencies. Near $1.3 \mathrm{GHz}$, the transfer functions (for IQ) of the klystrons can be approximated by a first order low pass with a $3 \mathrm{~dB}$ bandwidth of $3 \mathrm{MHz}$ :

$$
H_{\mathrm{kly}}(s)=\frac{1}{1+\frac{s}{2 \pi f_{3 \mathrm{~dB}}}} \text { with } f_{3 \mathrm{~dB}}=3 \mathrm{MHz} .
$$


The cavity transfer function is composed of the superposition of all nine transfer functions for the FMs described in the last section considering variations in the coupling (3) and loaded quality factors (4):

$$
\begin{gathered}
H_{(n / 9) \pi}(s) \stackrel{n \neq 9}{=}(-1)^{n+1} 2 \sin ^{2} \frac{n \pi}{18} \frac{\omega_{1 / 2}\left(s+\omega_{1 / 2}\right)}{\Delta \omega^{2}+\left(s+\omega_{1 / 2}\right)^{2}} \\
H_{\pi}(s)=\frac{1}{1+\frac{s}{\omega_{1 / 2}}}
\end{gathered}
$$

with

$$
\omega_{1 / 2}=\frac{\pi f_{(n / 9) \pi}}{Q_{L(n / 9) \pi}} \stackrel{n \neq 9}{=} 2 \sin ^{2} \frac{n \pi}{18} \frac{\pi f_{(n / 9) \pi}}{Q_{L \pi}}
$$

and ideal tuning of the $\pi$-mode

$$
\Delta \omega=2 \pi\left(f_{(n / 9) \pi}-f_{\pi}\right) .
$$

The factor $(-1)^{n+1}$ takes into account that alternate FMs have an electrical field in the end cell with opposite sign (Fig. 2) resulting in opposite sign between power coupler and pickup. Electrical voltage amplitudes couple like the square root of the coupling factor $\beta$. Both the coupling at the power coupler and the pickup results with (3) in the second factor in (5). For a derivation of the last factor see [1]. Summing up all nine transfer functions gives the cavity transfer function

$$
H_{\text {cav }}(s)=\sum_{n=1}^{9} H_{(n / 9) \pi}(s) .
$$

Because of the signal propagation time through cables, wave guides, high power rf, low power rf e.g. down converters, analog to digital converters (ADCs), field programmable gate arrays (FPGAs) and digital signal processors (DSPs), respectively, digital to analog converters (DACs) and as well through the control loop algorithms a certain loop delay $\tau_{\text {loop }}$ accumulates. Its transfer function is

$$
H_{\text {delay }}(s)=e^{-s \tau_{\text {loop }}}
$$

The loop delay and the loop gain

$$
H_{\text {gain }}(s)=G
$$

determine the loop stability.

To investigate the stability one examines how a high frequency error signal propagates through the open loop transfer function

$$
H(s)=H_{\text {gain }}(s) H_{\text {delay }}(s) H_{\text {cav }}(s) H_{\text {kly }}(s) .
$$

In case this error signal is phase shifted by more than $180 \mathrm{deg}$ and amplified (amplitude response $>0 \mathrm{~dB}$ ), one gets positive feedback and the closed loop becomes unstable. By drawing Bode plots of the open loop transfer function $[13,14]$, one can usually check visually whether this is the case.
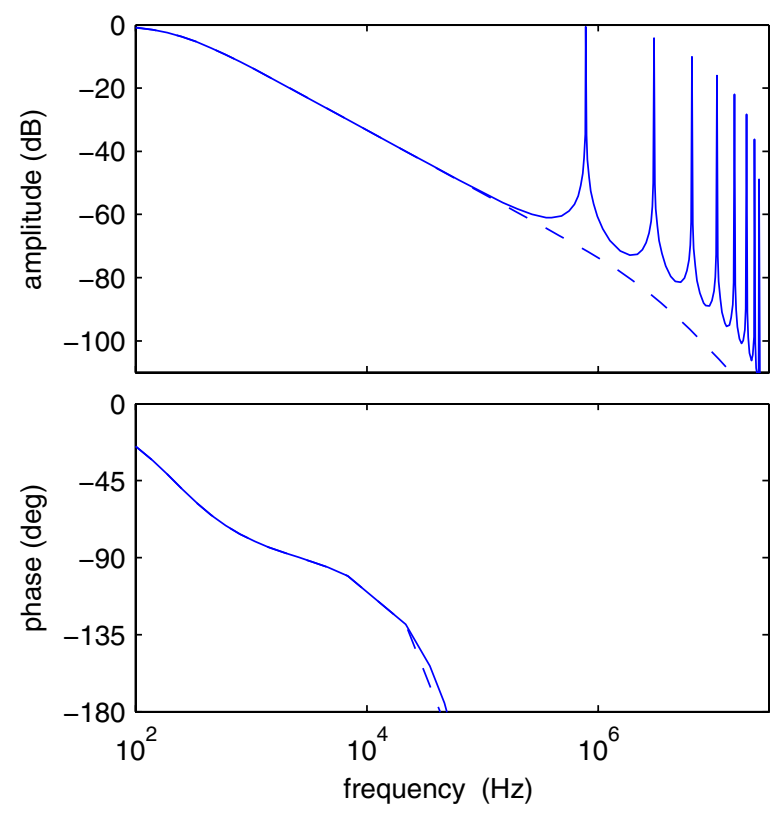

FIG. 4. (Color) Bode plot of the continuous single cavity open loop transfer function (7) with unity gain $(G=1)$ and loop delay $\tau_{\text {loop }}=5 \mu \mathrm{s}$. At the dashed line all FMs with exception of the $\pi$-mode have been omitted.

Plotting the amplitude and phase response of the open loop transfer function

$$
|H(i 2 \pi f)| \quad \text { and } \quad \arg H(i 2 \pi f)
$$

for unity gain $G=1$ and loop delay $\tau_{\text {loop }}=5 \mu$ s in logarithmic scales, one obtains the Bode plot Fig. 4.

Increasing the gain value moves the amplitude scale (Fig. 4) down. Hence, using the stability criterion from above, one reads from the Bode plot the maximum stable gain is about $L=46 \mathrm{~dB}$ and $G=200$ respectively ( $L=$ $20 \log _{10} G \mathrm{~dB}$ ) in case one omits all FMs with the exception of the $\pi$-mode (dashed line). The maximum transmission value of the $\frac{8}{9} \pi$-mode (Fig. 4 ) is about $-0.5 \mathrm{~dB}$, preventing any loop gain increase. For the maximum transmission values of the other modes, see Table III.

TABLE III. List of the FM peak levels $A$ for a continuous single cavity control with unity gain (values from Fig. 4).

\begin{tabular}{cc}
\hline \hline FM & $A$ \\
\hline$\pi$ & $0 \mathrm{~dB}$ \\
$8 / 9 \pi$ & $-0.5 \mathrm{~dB}$ \\
$7 / 9 \pi$ & $-4.1 \mathrm{~dB}$ \\
$6 / 9 \pi$ & $-10.0 \mathrm{~dB}$ \\
$5 / 9 \pi$ & $-15.9 \mathrm{~dB}$ \\
$4 / 9 \pi$ & $-21.8 \mathrm{~dB}$ \\
$3 / 9 \pi$ & $-28.2 \mathrm{~dB}$ \\
$2 / 9 \pi$ & $-36.2 \mathrm{~dB}$ \\
$1 / 9 \pi$ & $-48.8 \mathrm{~dB}$ \\
\hline \hline
\end{tabular}


At linear accelerators such as the ILC or the FELs cavities are driven by a klystron in groups of 8,16 , or even 32 [16] rather than singly and the sum of the angular pointers (vector sum) is controlled [1]. Because of production variation, each cavity has slightly different values for the difference frequencies $f_{(n / 9) \pi}-f_{\pi}$ resulting in more stable control loops as already remarked in [1]: At the $\frac{8}{9} \pi$-mode the rms variation of $50 \mathrm{kHz}$ (Table I) is much larger than the bandwidth of $420 \mathrm{~Hz}$. In the Bode plot of a vector sum control, the $\frac{8}{9} \pi$-mode lines appear for each cavity separately. Each time one doubles the number of cavities the maximum transmission values of the $\frac{8}{9} \pi$-modes are reduced by $6 \mathrm{~dB}$. The same is valid for the other modes. Hence, a continuous proportional vector sum control of eight cavities would be stable to gains up to $18 \mathrm{~dB}(G=8)$.

Digital proportional vector sum control loops are operated with higher gains as certain sampling schemes further attenuate the $\frac{8}{9} \pi$-mode and its companions. In the next sections we will discuss this mechanism.

\section{DIGITAL CONTROL WITH 1 MHZ SAMPLING}

A superconducting cavity with loaded quality factor of $Q_{L \pi} \approx 3 \times 10^{6}$ has a time constant of

$$
\tau_{\pi}=\frac{1}{\omega_{1 / 2}}=\frac{Q_{L \pi}}{\pi f_{\pi}} \approx 730 \mu \mathrm{s}
$$

Hence, sampling the amplitude and phase every $1 \mu \mathrm{s}$ (sampling rate of $1 \mathrm{MHz}$ ) is sufficiently fast for determining unwanted changes and feeding back control signals via a digital controller.

Current FPGA based rf field control at the free electron laser in Hamburg (FLASH) [16] samples the $250 \mathrm{kHz}$ intermediate frequency obtained after mixing the $1.3 \mathrm{GHz}$ cavity pickup signal with a $1.3 \mathrm{GHz}+250 \mathrm{kHz}$ reference signal [1] instead of sampling amplitude and phase. As a result a series of samples is obtained representing the components $I, Q,-I,-Q$ of the angular pointer characterizing the $1.3 \mathrm{GHz}$ cavity pickup signal (Fig. 5). Every $\mu \mathrm{s}$, an algorithm calculates the pointer components $I$ and $Q$ from the four samples previously taken and provides them to the subsequent control algorithms [17].

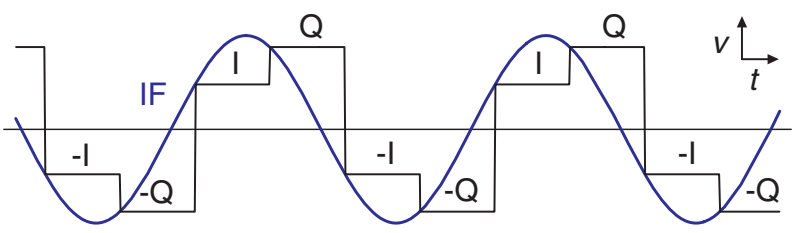

FIG. 5. (Color) Scheme of the "digital IQ detection," presently used at FLASH: Sampling the intermediate frequency with a four times higher sampling rate yields the vector components of the angular pointer with positive and negative sign.

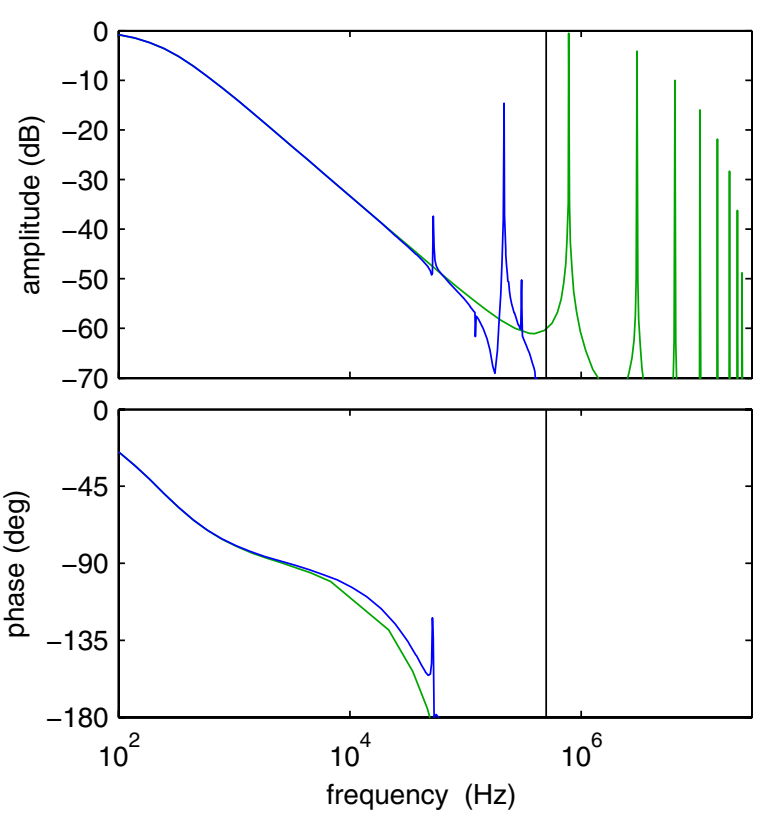

FIG. 6. (Color) Bode plot of the discrete single cavity open loop transfer function (blue) for $1 \mathrm{MHz}$ sampling rate, unity gain $G=$ 1 , and loop delay $\tau_{\text {loop }}=5 \mu \mathrm{s}$. The $\frac{8}{9} \pi$-mode maximum transmission value is aliased from 785 to $215 \mathrm{kHz}$ and reduced to a level of $-14.6 \mathrm{~dB}$. For comparison, the bode plot of the continuous system is also shown (green).

This approach results in an effective IQ sampling with $500 \mathrm{kHz}$, averaging subsequent samples at $500 \mathrm{kHz}$ and resampling the averaged values with $1 \mathrm{MHz}$. The IQ signals are delayed by $4 \mu \mathrm{s}$ due to this algorithm. Together with delays caused by cables, high power rf, and the control algorithms, the overall loop delay adds up to a value of $5 \mu \mathrm{s}$. For error correction a DAC, also operating at $1 \mathrm{MHz}$, steers the rf drive signal via a vector modulator (Fig. 3).

With a sample rate of $1 \mathrm{MHz}$ frequencies up to one-half of the sample frequency, namely, $500 \mathrm{kHz}$ can be represented. Consequently, the Bode plot of the discrete transfer function covers only frequencies from zero to $500 \mathrm{kHz}$. Nevertheless, the unwanted FMs still play an important role for the loop stability even when their frequencies differ by more than $500 \mathrm{kHz}$ from the $\pi$-mode frequency.

Assume a $200 \mathrm{kHz}$ signal generated with a DAC by switching the DACs output every $\mu$ s appropriately (zero order hold). The resulting analog signal consists mainly of a $200 \mathrm{kHz}$ signal but also of higher harmonic signals with $400 \mathrm{kHz}, 600 \mathrm{kHz}, 800 \mathrm{kHz}$, and so on, due to the steps caused by the switching. Compared to the $200 \mathrm{kHz}$ amplitude, the $800 \mathrm{kHz}$ amplitude is attenuated by $12.05 \mathrm{~dB}$. In the case of a cavity whose $\frac{8}{9} \pi$-mode is exactly $800 \mathrm{kHz}$ away from the $\pi$-mode, the $\frac{8}{9} \pi$-mode will be driven by the $200 \mathrm{kHz}$ signal but with an input amplitude attenuated by $12.05 \mathrm{~dB}$. Sampling $800 \mathrm{kHz}$ with $1 \mathrm{MHz}$ results in an alias frequency of $200 \mathrm{kHz}$ whose amplitude is attenuated by 
$0.58 \mathrm{~dB}$ with respect to the $800 \mathrm{kHz}$ signal. The filter characteristic of the sampling scheme (averaging and resampling) is included when the Bode plot of the discrete transfer function with $1 \mathrm{MHz}$ sampling rate shows the line of the $\frac{8}{9} \pi$-mode at $200 \mathrm{kHz}$ attenuated by $14 \mathrm{~dB}$ with respect to the continuous transfer function. A similar argument is valid for the other FMs.

Using the "c2d" command within MATLAB [18], one may perform the examination described for all frequencies automatically and directly generate the Bode plot of the discrete system (Fig. 6). An analytical method is described in [19]. Sampling at $1 \mathrm{MHz}$ already attenuates the amplitude response of the second strongest FM ( $\frac{7}{9} \pi$-mode) to a level of $-37.3 \mathrm{~dB}$.

In the previous section, attenuation of FM lines by $18 \mathrm{~dB}$ due to an eight cavity vector sum control has been discussed. Together with $1 \mathrm{MHz}$ sampling, one can increase the gain to $32 \mathrm{~dB}(G=40)$ until one gets an instability due to the $\frac{8}{9} \pi$-mode. The next highest FM shows a maximum level of $55 \mathrm{~dB}(G=562)$ and is therefore of no further interest since the $\pi$-mode already becomes unstable for $G \approx 200$ with $\tau_{\text {loop }}=5 \mu \mathrm{s}$.

\section{STABILITY CHART FOR 1 MHZ SAMPLING}

According to the results of the previous section, digital control with $1 \mathrm{MHz}$ attenuates all unwanted FMs to levels irrelevant for the loop stability with the exception of the $\frac{8}{9} \pi$-mode. Therefore, this section presents loop stability examinations of the continuous transfer function describing the cavity hosting the $\pi$-mode and $\frac{8}{9} \pi$-mode only.

For simplicity, attenuation of the $\frac{8}{9} \pi$-mode by both the sampling and the restricted klystron bandwidth will be neglected resulting in a worst case estimate.

The stability of the simplified transfer function

$$
H(s)=G\left(H_{\pi}(s)+H_{(8 / 9) \pi}(s)\right) e^{-s \tau_{\text {loop }}}
$$

can be examined in more detail using the Nyquist stability criterion.

Assuming a control loop with the open loop transfer function $H(s)$ as in Fig. 7, the Nyquist diagram is obtained by plotting successive values of $H(i \omega)$ as a function of the parameter $\omega$, running from zero to infinity, in the complex, so-called $H(s)$-plane. For the resultant diagram the Nyquist theorem says [14]: The closed-loop system is stable if and only if the number of clockwise encirclements of the point $s=-1+i 0$ by the Nyquist diagram of $H(s)$ plus the number of poles of $H(s)$ in the right half-plane is zero.

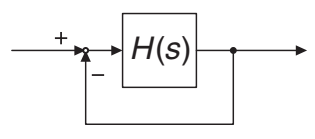

FIG. 7. Control loop with open loop transfer function $H(s)$.

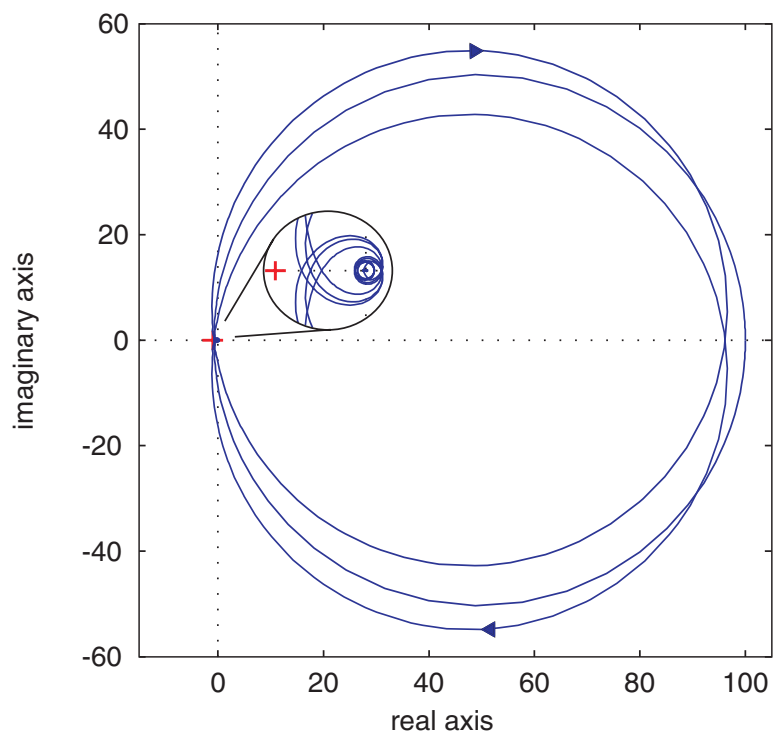

FIG. 8. (Color) Nyquist diagram for the open loop transfer function of a cavity hosting the $\pi$-mode and $\frac{8}{9} \pi$-mode only with $\tau_{\text {loop }}=5.6 \mu$ s and $G=100$. The circle contains a zoomed view of the region around the origin and the point $s=-1+i 0$ (cross).

If one generates the Nyquist diagram of the transfer function (8) with a loop delay of $\tau_{\text {loop }}=5.6 \mu \mathrm{s}$ and a gain of $G=100$ (Fig. 8), one realizes the loop would be stable. According to the stability examinations performed until now, one would expect an unstable behavior because the $\frac{8}{9} \pi$-mode causes a line above $0 \mathrm{~dB}$ after the phase of the transfer function has already passed $180 \mathrm{deg}$. This contradiction can be resolved as follows.

For zero loop delay the $\frac{8}{9} \pi$-mode has an opposite field amplitude sign in the end cell as compared to the $\pi$-mode (Fig. 2) resulting in positive feedback and an unstable loop at higher gain values. A loop delay of $\tau_{\text {loop }}=0.62 \mu \mathrm{s}$ changes the phasing of the $\frac{8}{9} \pi$-mode by about $180 \mathrm{deg}$ resulting in a negative feedback and a stable loop. Hence, increasing the loop delay further, the phasing of the $\frac{8}{9} \pi$-mode changes with a period of $1 /\left(f_{(8 / 9) \pi}-f_{\pi}\right)$ resulting in subsequent regions where the loop is unstable and stable. For the loop delay plotted (Fig. 8), the phase advance is about 4.5 times $360 \mathrm{deg}$ which is equivalent to $180 \mathrm{deg}$ and therefore results in a stable loop.

Inspecting Nyquist diagrams for various loop delays and gains results in the stability chart shown in Fig. 9. In reality, the stable areas are larger due to the effects discussed in the previous sections, such as the spread in the frequencies of the $\frac{8}{9} \pi$-modes at a vector sum control of eight or more cavities and the attenuation through the sampling.

From this stability chart, one can determine settings for loop delays and gain values for which a single cavity proportional $\mathrm{rf}$ control loop operating at $1 \mathrm{MHz}$ sample rate would be stable. In practice, if the loop delay results in an unstable operation point one may additionally delay the 


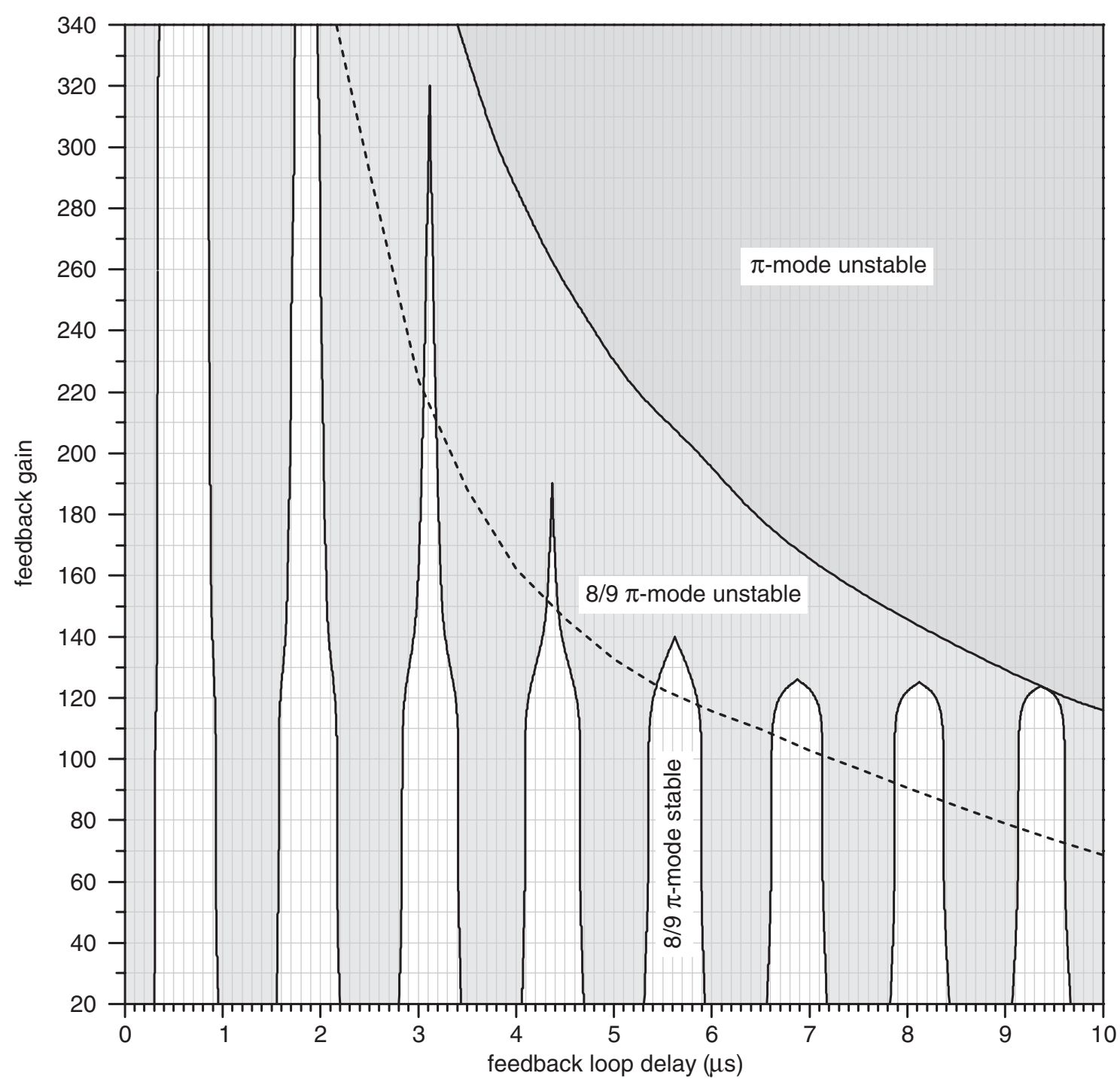

FIG. 9. Stability chart for a single cavity digital control loop operating with $1 \mathrm{MHz}$ sample rate. For simplicity, $800 \mathrm{kHz}$ was chosen for the $\frac{8}{9} \pi$-mode difference frequency and equal coupling for both modes $\left(\beta_{(8 / 9) \pi}=\beta_{\pi}\right)$. In practice, the stable areas are more expanded, see text. A phase margin larger than $30 \mathrm{deg}$ with respect to the $\pi$-mode is obtained by choosing gain values below the dashed line.

output signal of the controller to reach a stable area. Hence, inserting an additional delay, one may be able to overcome a gain limit of $32 \mathrm{~dB}(G=40)$ at an eight cavity control.

\section{CONTROL WITH 54 MHZ SAMPLING}

A superconducting TESLA cavity is an extreme narrow band filter. The rf noise coming from the vector modulator and the high power $\mathrm{rf}$ is massively suppressed. As a result the cavity field contains very little noise. The noise within the pickup signal measured is dominated by noise picked up between the cavity pickup antenna and the ADC. Sampling the signal at high frequencies enables averaging and filtering to improve the signal to noise ratio. For white noise, sampling $N$ times the signal and taking the average increases the signal to noise ratio by $\sqrt{N}$.
Decimation and filtering will be neglected in this section and discussed later. The consequences of choosing a high sample frequency only are discussed first.

Within the development process towards an rf control system for the European XFEL [16], the sampling of a 13.5 MHz intermediate frequency $(1300 \mathrm{MHz} / 96)$ with $54 \mathrm{MHz}(1300 \mathrm{MHz} / 24)$ is planned as one of the next steps [17]. Each $18.5 \mathrm{~ns}$, the angular pointer components $I$ and $Q$ can be calculated from the four samples previously taken resulting in a minimum delay of $74 \mathrm{~ns}$. The loop delay will be reduced to a value around $1 \mu \mathrm{s}$.

Figure 10 shows the Bode plot for the transfer function (7) under the effect of sampling with $54 \mathrm{MHz}$ and a loop delay of $\tau_{\text {loop }}=1 \mu \mathrm{s}$. Attenuation of the unwanted modes by the sampling becomes negligible due to the high sample frequency. As a result, the stable areas in the stability chart 


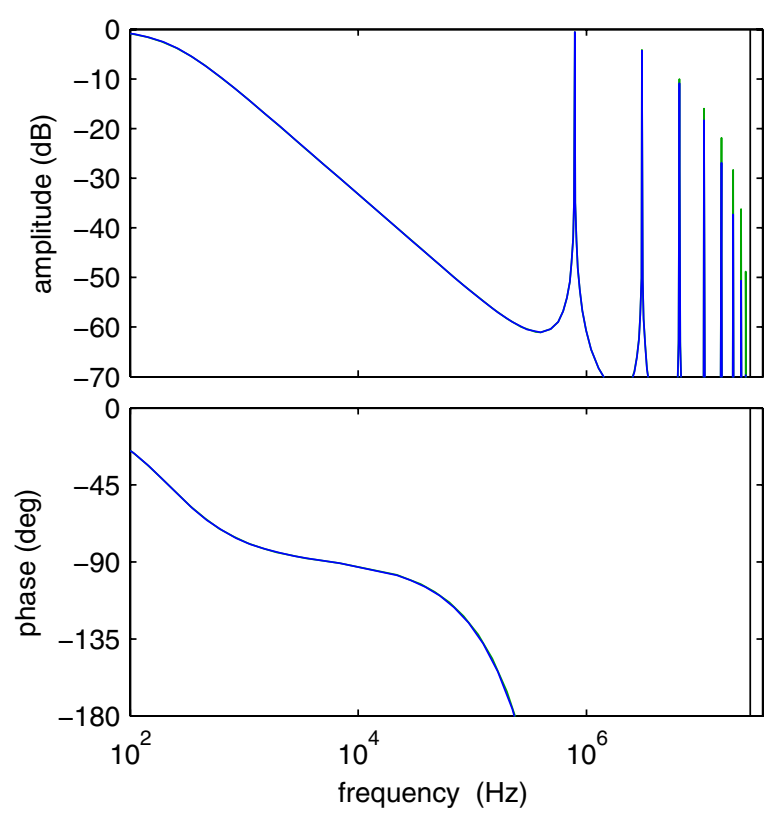

FIG. 10. (Color) Bode plot of the discrete single cavity open loop transfer function (blue) for $54 \mathrm{MHz}$ sampling rate, unity gain $G=1$, and loop delay $\tau_{\text {loop }}=1 \mu \mathrm{s}$. For comparison, the bode plot of the continuous system is also shown (green). The digitalization attenuates the $\frac{8}{9} \pi$-mode, the $\frac{7}{9} \pi$-mode, and the $\frac{6}{9} \pi$-mode only weakly, the other modes are more attenuated.

(Fig. 9) are subdivided by unstable and stable areas for the $\frac{7}{9} \pi$-mode, $\frac{6}{9} \pi$-mode, and so on. Ensuring the control loop stability by increasing the loop delay becomes more delicate because the remaining stable areas are much smaller.

The disadvantage of little or no attenuation due to the sampling can be compensated by the addition of digital filters working at high sample frequencies. A "brute force" method may be the implementation of a digital low pass with a steep slope between 500 and $800 \mathrm{kHz}$ attenuating all unwanted FMs. This would also restrict the bandwidth of the rf control and therefore reduce the speed for error correction. Rather than including a low pass, the use of well adjusted digital notch filters will attenuate the unwanted FMs and not restrict the bandwidth of the regulation.

Delaying a signal sampled with $f_{\mathrm{s}}=54.2 \mathrm{MH} z$ by 34 sample steps and adding the value to the actual one (Fig. 11) results in the $z$-transfer function

$$
H_{\mathrm{nf}}(z)=\frac{1}{2}\left(1+z^{-34}\right)
$$

where the amplitude response is given by $\left|H_{\mathrm{nf}}(i \Omega)\right|$ with $\Omega=\omega / f_{\mathrm{s}}$. Performing some algebra

$$
\begin{aligned}
H_{\mathrm{nf}}(i \Omega) & =\frac{1}{2}\left(1+e^{-34 i \Omega}\right)=\frac{1}{2} e^{-17 i \Omega}\left(e^{17 i \Omega}+e^{-17 i \Omega}\right) \\
& =e^{-17 i \Omega} \cos 17 \Omega,
\end{aligned}
$$

the absolute value and amplitude response becomes

$$
\left|H_{\mathrm{nf}}(i \Omega)\right|=|\cos 17 \Omega|
$$

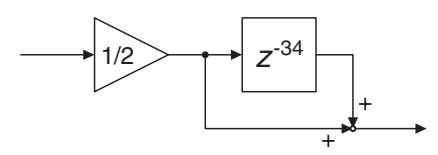

FIG. 11. Block diagram of a simple notch filter.

resulting in zero amplitude response for

$$
\begin{aligned}
17 \Omega & =34 \pi f / f_{\mathrm{s}}=\frac{\pi}{2}, 3 \frac{\pi}{2}, 5 \frac{\pi}{2}, \ldots \\
f & =797 \mathrm{kHz}, 2.39 \mathrm{MHz}, 3.99 \mathrm{MHz}, \ldots
\end{aligned}
$$

which is a notch filter with very steep flanks.

Because of the narrow bandwidth of the unwanted FMs, the notch filter frequency has to be properly matched with the mode frequency to be rejected. In the case of a $\frac{8}{9} \pi$-mode frequency of $f_{(8 / 9) \pi}-f_{\pi}=785 \mathrm{kHz}$, the notch frequency of $797 \mathrm{kHz}$ would be too high. The next lower notch filter frequency obtained by using a delay by 35 samples instead of 34 samples would lead to the lowest notch frequency at $774 \mathrm{kHz}$, which also does not match.

Inspired by [20,21], a weighted combination of two notch filters also results in a notch filter at a frequency which can be chosen freely (Fig. 12): To create a notch filter for $f_{\text {wnf }}$, one determines the sample delay value resulting in the next notch filter frequencies above the frequency $f_{\text {wnf }}$

$$
N=\text { integer part } \frac{f_{\mathrm{s}}}{2 f_{\mathrm{wnf}}},
$$

adding one gives the next notch filter frequency below the frequency $f_{\text {wnf }}$. The weights of the two filters are given by

$$
w=\text { fractional part } \frac{f_{\mathrm{s}}}{2 f_{\mathrm{wnf}}},
$$

resulting in the filter transfer function for the combination

$$
H_{\mathrm{wnf}}(z)=\frac{w}{2}\left(1+z^{-N-1}\right)+\frac{1-w}{2}\left(1+z^{-N}\right) .
$$

Table IV contains the numerical values of the notch filter parameters for the first four unwanted FMs and their maximum attenuation. For higher notch frequencies the difference frequency between the two parts of the combined notch filter becomes larger and the resulting filter less effective. As an example, the notch filter for the

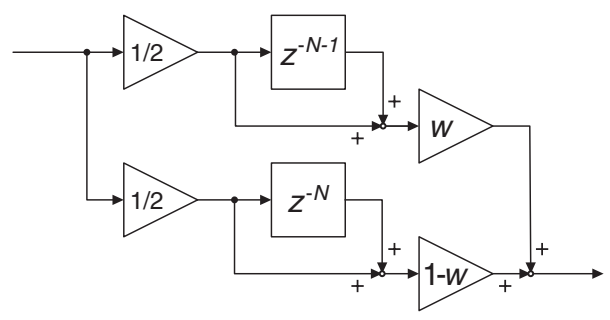

FIG. 12. Block diagram of a combined notch filter whose frequency can be chosen freely. 
TABLE IV. List of notch filter parameters calculated for a sample frequency of $54 \mathrm{MHz}$ and the maximum attenuation $A$ obtained.

\begin{tabular}{crrrr}
\hline \hline FM & \multicolumn{1}{c}{$f_{\text {wnf }}$} & \multicolumn{1}{c}{$N$} & \multicolumn{1}{c}{$w$} & \multicolumn{1}{c}{$A$} \\
\hline $8 / 9 \pi$ & $784640 \mathrm{~Hz}$ & 34 & 0.5169 & $-65.7 \mathrm{~dB}$ \\
$7 / 9 \pi$ & $3052747 \mathrm{~Hz}$ & 8 & 0.8718 & $-49.1 \mathrm{~dB}$ \\
$6 / 9 \pi$ & $6500872 \mathrm{~Hz}$ & 4 & 0.1661 & $-34.2 \mathrm{~dB}$ \\
$5 / 9 \pi$ & $10693844 \mathrm{~Hz}$ & 2 & 0.5326 & $-20.7 \mathrm{~dB}$ \\
\hline \hline
\end{tabular}

$\frac{5}{9} \pi$-mode with $f_{(5 / 9) \pi}-f_{\pi}=10.7 \mathrm{MHz}$ would consist of the two notch filters at $9 \mathrm{MHz}$ and at $13.6 \mathrm{MHz}$ attenuating the $10.7 \mathrm{MHz}$ by $20.7 \mathrm{~dB}$. This is much lower than that achieved by the notch filter for the $\frac{8}{9} \pi$-mode which attenuates $785 \mathrm{kHz}$ by $65.7 \mathrm{~dB}$.

For the suppression of higher FM frequencies the use of an 18 tap FIR low pass filter with a lower band edge frequency of $8 \mathrm{MHz}$ is suitable

$$
H_{\mathrm{FIR}}(z)=\sum_{\mu=1}^{18} h_{\mu} z^{-\mu}
$$

The filter parameters $h_{\mu}$ are given in Table V. This low pass does not restrict the bandwidth of the rf control and the reasonable number of 18 taps is sufficient.

The extra delay of $1 \mu$ s caused by three subsequent notch filters for the $\frac{8}{9} \pi$-mode, the $\frac{7}{9} \pi$-mode, and the $\frac{6}{9} \pi$-mode and the FIR low pass filter increases the overall loop delay to $\tau_{\text {loop }}=2 \mu \mathrm{s}$. Taking this into account, the Bode plot for a single cavity control applying the filters described can be drawn (Fig. 13).

If a notch filter does not exactly match an unwanted FM, the maximum FM transmission value grows. Figure 13 shows the situation, when all notch filters have a difference frequency of $500 \mathrm{~Hz}$ to the FMs which might be caused by static tuning and dynamic detuning effects as caused by the Lorentz force. Even when the notch filters are off by $5 \mathrm{kHz}$, all FMs will be attenuated to levels below $-41.7 \mathrm{~dB}$ which would still allow for a gain of $59 \mathrm{~dB}(G=891)$ at an eight cavity vector sum control. Nevertheless, the filters need to be adjusted to the individual cavities due to the spread in the FM difference frequencies of $50 \mathrm{kHz}$ and more (Table I).

First simulation of the pulsed rf operation case shows that the signals can be notch filtered directly after sampling and in front of the error signal calculation. Hence, for vector sum control the FMs may be filtered by notch filters, adjusted for each cavity individually, before calculating the vector sum and the error signal.

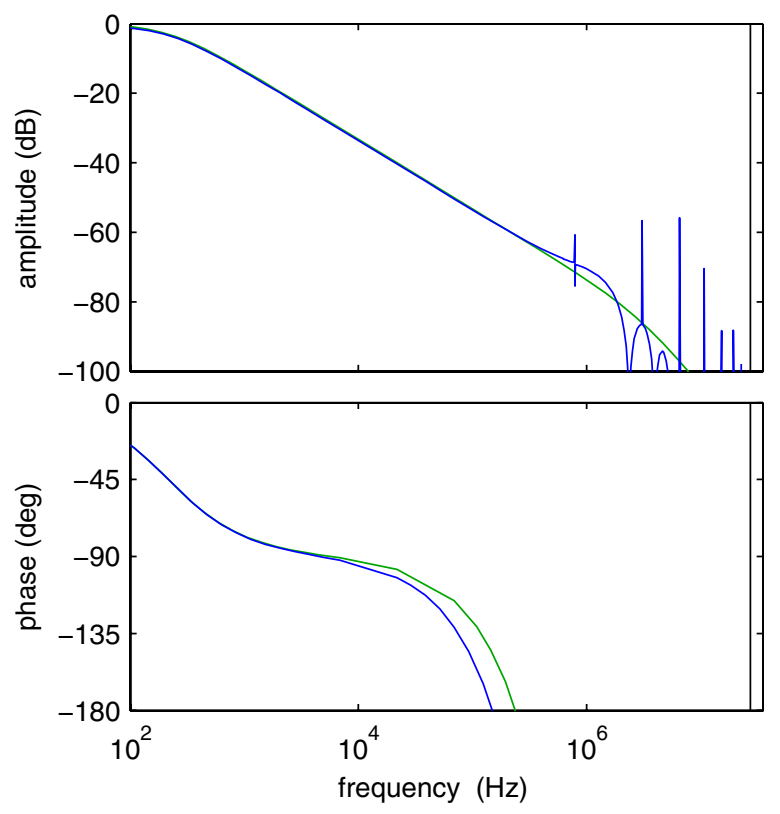

FIG. 13. (Color) Bode plot of the discrete single cavity open loop transfer function (blue) for $54 \mathrm{MHz}$ sampling rate, unity gain $G=1$, and loop delay $\tau_{\text {loop }}=2 \mu \mathrm{s}$. Notch filters are applied to the $\frac{8}{9} \pi$-mode, the $\frac{7}{9} \pi$-mode, and the $\frac{6}{9} \pi$-mode together with an $8 \mathrm{MHz}$ FIR low pass filter. For comparison, the bode plot of the transfer function containing a cavity with $\pi$-mode only is also shown (green).

\section{AVERAGING AND DECIMATION}

Averaging increases the signal to noise ratio as discussed in the previous section. If one uses a moving average retaining the sample frequency, the averaging causes an additional delay of one-half of the number of samples averaged. This additional delay is the only effect on the loop stability.

Calculating the average value of $N$ samples and passing only the average value with $N$ times lower rate to the subsequent algorithms (decimation) causes an additional delay by $N$ samples (with the high rate). As the DAC is now operating with the lower sample frequency, frequencies are aliased to frequencies from zero to one-half of this frequency. The aliased frequencies are attenuated by the zero order hold as discussed in the section treating the $1 \mathrm{MHz}$ sampling.

When one samples with $54 \mathrm{MHz}$ and decimates the signals to $1 \mathrm{MHz}$ at the DAC, the $\frac{8}{9} \pi$-mode is attenuated by $12 \mathrm{~dB}$ resulting in approximately the same stability as discussed for $1 \mathrm{MHz}$ sampling. Using such a scheme, notch

TABLE V. Coefficients of an $8 \mathrm{MHz}$ FIR low pass with a sample frequency of $54 \mathrm{MHz}$ and an attenuation of $-28 \mathrm{~dB}$.

\begin{tabular}{ccccccccc}
\hline \hline$h_{1}=h_{18}$ & $h_{2}=h_{17}$ & $h_{3}=h_{16}$ & $h_{4}=h_{15}$ & $h_{5}=h_{14}$ & $h_{6}=h_{13}$ & $h_{7}=h_{12}$ & $h_{8}=h_{11}$ & $h_{9}=h_{10}$ \\
\hline 0.0079 & -0.0248 & -0.0274 & -0.0218 & 0.0043 & 0.0525 & 0.1137 & 0.1708 & 0.2053 \\
\hline \hline
\end{tabular}


filtering of the unwanted FMs becomes unnecessary and choosing a suitable loop delay is sufficient.

The maximum bunch repetition frequency of the European XFEL will be $5 \mathrm{MHz}$. Beam based rf feedback loops may be operated in the ideal case with this frequency resulting in a DAC sample rate of $5 \mathrm{MHz}$ rather than $1 \mathrm{MHz}$. The $\frac{8}{9} \pi$-mode would be much less attenuated by the zero order hold. The use of notch filters directly after the ADCs operating at high sample frequency before the decimation would avoid instabilities.

\section{DAMPING OF FUNDAMENTAL MODES}

The previous sections treated the stability of high gain proportional rf control and described measures facilitating a significant increase of the loop gain to values larger than $40 \mathrm{~dB}(G=100)$. Attenuating the unwanted FM frequencies by filters results in a stable control loop, but the FMs are still driven causing small oscillations of amplitude and phase. Simulation including the notch filters described shows these oscillations may have amplitudes of the order of the rf stability requirements for the European XFEL of $\pm 0.01 \mathrm{deg}$ [16]. They are not present in the case of a loop delay adjustment resulting in a negative feedback for the $\frac{8}{9} \pi$-mode. With high frequency sampling ( $54 \mathrm{MHz}$ ), a loop delay adjustment resulting in a negative feedback for all unwanted FMs is impractical. The unwanted FM frequencies can be damped as follows.

Subtracting the output signal of a notch filter from its input signal gives the signal attenuated by the notch. Hence, in the case of the use of a notch filter adjusted for the $\frac{8}{9} \pi$-mode, one obtains the signal of the $\frac{8}{9} \pi$-mode only. Depending on the signal propagation time and taking the phasing in the end cells (Fig. 2) into account, one may add or subtract this signal after amplification to the feedback correction signal (Fig. 14). As a result the modes are damped. Furthermore, instead of changing only the sign, the loop phase for such an FM damping loop may also be adjusted for optimal performance. This can be done inde-

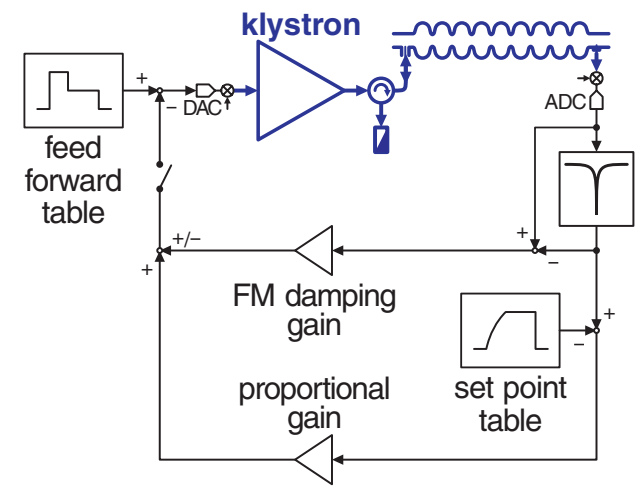

FIG. 14. (Color) Sketch of a pulsed rf superconducting cavity control loop with damping of an unwanted FM. pendently for each FM damping loop and independent from the rf control loop.

Simulation containing cavities with $\pi$-mode and $\frac{8}{9} \pi$-mode and applying notch filtering together with the $\frac{8}{9} \pi$-mode damping show quite promising results. The remaining phase oscillations caused by driving the $\frac{8}{9} \pi$-mode can be damped to levels well below the XFEL stability requirements.

\section{INTEGRAL CONTROL}

Integrating the error signals over a sufficiently long period of time and feeding the resultant value back suppresses the effect of the unwanted FMs immediately. Hence, by the use of an integral control instead of a proportional control one can realize high gain error suppression for low frequency errors with much less effort. This does not result in high gain values for the suppression of high frequency errors discussed here. Furthermore, the use of a proportional together with an integral control (PI control) does not result in more gain margin for the proportional control.

\section{EXPERIMENTAL VERIFICATION}

When TESLA cavities were controlled for the first time in 1996 by an rf control system based on DSPs, the stability could be improved by prolonging the loop delay so that the $\frac{8}{9} \pi$-mode was negatively fed back and damped rather than amplified [22]. These rf control systems were based on the sampling of a $250 \mathrm{kHz}$ intermediate frequency with $1 \mathrm{MHz}$ which is in correspondence to the situation discussed.

The first accelerating module at FLASH, consisting of eight cavities, is controlled by a FPGA based rf field control with a loop delay of $5 \mu \mathrm{s}$ and a sampling rate of $1 \mathrm{MHz}$. Increasing the loop gain to values above 30 $(\approx 30 \mathrm{~dB})$ results in an unstable behavior [17]. This is in good agreement with the attenuation of the $\frac{8}{9} \pi$-mode effect by $32 \mathrm{~dB}$ composed of $14 \mathrm{~dB}$ due to the sampling and $18 \mathrm{~dB}$ for the case that eight cavities are controlled with a loop delay leading to an operating point in an unstable area (Fig. 9).

First measurements examining the loop stability as a function of the loop delay in the case of $1 \mathrm{MHz}$ sampling were performed. A periodic sequence with a period of $1.3 \mu \mathrm{s}$ in the loop delay was observed where the loop became subsequently stable and unstable [23]. This fits to the expectations as the period observed corresponds to the difference frequency of the $\frac{8}{9} \pi$-mode.

\section{SUMMARY AND OUTLOOK}

Proportional control is the basis for the rf field stabilization in TESLA cavities. These cavities consist of nine cells and therefore host nine fundamental modes (FMs) of which only one ( $\pi$-mode) is desired for acceleration. The paper 
contains a derivation of relevant mode parameters such as the coupling and the loaded quality factors together with frequency values measured.

A continuous proportional single cavity rf control without special filters would allow for a maximum stable gain of $0.5 \mathrm{~dB}(G=1.1)$ due to the unwanted FMs even when one takes into account the bandwidth limitation of the klystron. Production differences lead to a spread of the frequency differences between the unwanted FMs and the $\pi$-mode larger than the mode bandwidth. Hence, each doubling of the number of cavities in a single klystron multicavity control (vector sum control) leads to a doubling of the gain margin. With eight cavities, the maximum stable gain would be $18 \mathrm{~dB}(G=7.9)$.

The use of a digital controller operating with $1 \mathrm{MHz}$ sample rate aliases the FM difference frequencies into the frequency range from zero to $500 \mathrm{kHz}$ and attenuates the maximum transmission value of the $\frac{8}{9} \pi$-mode by $14 \mathrm{~dB}$ and the maximum transmission values of the other unwanted FMs to safe levels. Depending on the loop delay the $\frac{8}{9} \pi$-mode is fed back positively or negatively. In case of a negative feedback the $\frac{8}{9} \pi$-mode is damped and high gain values above $40 \mathrm{~dB}(G>100)$ are facilitated.

Higher sample frequencies such as $54 \mathrm{MHz}$ at the ADCs and DACs have little or no attenuation effect on the unwanted FMs. The use of digital notch filters and a digital low pass not restricting the loop bandwidth is feasible. For optimal attenuation the filters can be adjusted for each cavity separately. As a result, high gain values above $40 \mathrm{~dB}(G>100)$ are possible. In addition, potentially remaining small oscillations caused by FMs without danger for the loop stability but spoiling the rf field quality at the $10^{-4}$ level may be damped. Using decimation, the lower frequency and the frequency of the DAC determines the loop stability.

Observations from proportional rf control loops operating with $1 \mathrm{MHz}$ sample rate and regulating the rf fields at FLASH are in good agreement with the estimates presented.

The maximum gain values discussed can only be reached in the ideal case. Temperature changes in cables and electronics may cause loop phase variations. A klystron operated close to saturation also leads to phase distortions if not compensated. Neglecting cavity detuning effects, as done in this paper, is justified in the case piezo tuners are used and for small rf gradients. Otherwise the rf phases are affected. All these effects cause instabilities already at lower gain values than those discussed. Hence, in practice one operates with lower gain values to keep some margin in phase and amplitude and provide robustness against unwanted parameter variations. Typical values for phase margins are larger than $30 \mathrm{deg}$.

This paper may serve as a starting point for similar examinations for more sophisticated rf control methods such as optimal multi-input multi-output control [24,25] taking cavity detuning effects into account and for the elaboration of new sampling [26] and decimation schemes.

\section{ACKNOWLEDGMENTS}

I would like to thank Stefan Simrock for sharing with me his practical experience concerning rf control stability and the $\frac{8}{9} \pi$-mode. I am grateful to Paul-Dieter Gall and Vladimir Gubarev who provided the fundamental mode frequencies. Furthermore I would like to thank Jacek Sekutowicz for discussions on the coupling of the fundamental modes. With sincerest appreciation I would like to mention Vladimir Vogel for several meetings concerning the measured klystron frequency response. For discussions about technical details important for the loop stability and the next hardware development steps, I would like express my gratitude to Waldemar Koprek. For talks on various control theoretical aspects, I would like to thank Gerwald Lichtenberg and Christian Schmidt. I would also like to thank Alexander Brandt and Wojciech Jalmuzna for the collaboration at first examinations concerning the dependence of the loop stability on the loop delay. I am grateful to Maria Elena Angoletta for bringing the paper of Alexander Schnase et al. to my attention. I would like to express my gratitude to Gustavo Cancelo for discussions on the windowing effect due to IQ sample schemes. For further suggestions and careful proofreading, I would like to thank Reinhard Brinkmann and John Maidment.

[1] T. Schilcher, Ph.D. thesis, University of Hamburg, 1998 (TESLA Report No. TESLA 1998-20, 1998).

[2] R. Zhang, I. Ben-Zvi, and J. Xie, Nucl. Instrum. Methods Phys. Res., Sect. A 324, 421 (1993)

[3] M. Liepe, Diploma thesis, University of Hamburg, 1998.

[4] H. Piel, in Proceedings of the CERN Accelerator School: Superconductivity in Particle Accelerators, Hamburg, Germany, 1988 (CERN Report No. CERN-89-04, 1989).

[5] H. Ma, M. Champion, M. Crofford, K.-U. Kasemir, M. Piller, L. Doolittle, and A. Ratti, Phys. Rev. ST Accel. Beams 9, 032001 (2006).

[6] E. Bright Wilson, Jr., J. C. Decius, and Paul C. Cross, Molecular Vibrations-The Theory of Infrared and Raman Vibration Spectra (Dover Publications, Inc., New York, 1980).

[7] E. P. Wigner, Group Theory and its Application to the Quantum Mechanics of Atomic Spectra (Academic Press, New York and London, 1959).

[8] M. Wagner, Gruppentheoretische Methoden in der Physik. Ein Lehr- und Nachschlagewerk (Springer-Verlag, Berlin, 1998).

[9] P.-D. Gall and V. Gubarev (personal communication).

[10] C. Peschke, Diploma thesis, Johann Wolfgang GoeteUniversität Frankfurt am Main, 1995.

[11] R. Wanzenberg, TESLA Report No. TESLA 2001-33, 2001.

[12] J. Sekutowicz (personal communication). 
[13] H. Lutz and W. Wendt, Taschenbuch der Regelungstechnik (Verlag Harri Deutsch, Frankfurt am Main, 2002), 4., korrigierte Auflage.

[14] The Control Handbook, edited by W. S. Levine (CRC and IEEE Press, Boca Raton, FL, 1996).

[15] V. Vogel (personal communication).

[16] DESY Report DESY 2006-097, 2006, edited by Massimo Altarelli, Reinhard Brinkmann, Majed Chergui, Winfried Decking, Barry Dobson, Stefan Düsterer, Gerhard Grübel, Walter Graeff, Heinz Graafsma, Janos Hajdu, Jonathan Marangos, Joachim Pflüger, Harald Redlin, David Riley, Ian Robinson, Jörg Rossbach, Andreas Schwarz, Kai Tiedtke, Thomas Tschentscher, Ivan Vartaniants, Hubertus Wabnitz, Hans Weise, Riko Wichmann, Karl Witte, Andreas Wolf, Michael Wulff, and Mikhail Yurkov; http://xfel.desy.de/tdr/index_eng.html.

[17] W. Koprek (personal communication).

[18] A. Angermann, M. Beuschel, M. Rau, and U. Wohlfarth, Matlab-Simulink-Stateflow (Oldenbourg, VerlagMünchen-Wien, 2003), 2. überarbeitete Auflage; the
MATLAB Help browser provides similar information in English.

[19] G. F. Franklin, J. D. Powell, and M. Workman, Digital Control of Dynamic Systems (Addison-Wesley-Longman, Menlo Park, CA, 1998), 3rd ed.

[20] A. Schnase, M. Nomura, F. Tamura, M. Yamamoto, S. Anami, E. Ezura, K. Hara, C. Ohmori, A. Takagi, and M. Yoshii, Phys. Rev. ST Accel. Beams 8, 122001 (2005).

[21] E. B. Hogenauer, IEEE Trans. Acoust. Speech Signal Process., ASSP-29, 2 (1981).

[22] S. Simrock (personal communication).

[23] A. Brandt, W. Jalmuzna, and E. Vogel, unpublished examinations performed at FLASH, DESY (2006).

[24] D.E. Kirk, Optimal Control Theory: An Introduction (Dover Publications Inc., New York, 2004).

[25] G. Koch, Diploma thesis, Technical University of Hamburg-Harburg, 2005.

[26] M. Grecki, T. Jeśyński, and A. Brandt, in Proceedings of the 12th Mixed Design of Integrated Circuits and Systems, MIXDES 2005, Cracow, Poland, 2005, pp. 783-788. 